Psychotherapie aktuell

Psychotherapeut 2020 $65: 285-290$

https://doi.org/10.1007/s00278-020-00426-w

Online publiziert: 18 . Mai 2020

(c) Springer Medizin Verlag $\mathrm{GmbH}$, ein Teil von

Springer Nature 2020

\author{
Thomas H. Loew ${ }^{1} \cdot$ Maximilian Krinninger $^{2} \cdot$ Christian Kettler $^{3}$ \\ ${ }^{1}$ Abteilung für Psychosomatik, Universitätsklinikum Regensburg, Regensburg, Deutschland \\ ${ }^{2}$ Alpcura Fachklinik Allgäu Betriebsgesellschaft mbH, Pfronten, Deutschland \\ ${ }^{3}$ Fachklinik St. Lukas, Bad Griesbach, Deutschland
}

\title{
Stationäre Psychosomatik in Zeiten des Coronavirus
}

vonstattengehen wird, werden im Folgenden die zentralen Aspekte, die zum größten Teil hygienische Maßnahmen betreffen, zusammengestellt.

\section{Wie haben sich die Kliniken aktuell aufgestellt?}

Grundsätzlich können folgende 3 Versorgungsmuster und Angebote der Einrichtungen unterschieden werden:

1. Die psychosomatische Abteilung oder Klinik fährt komplett herunter, entlässt die Patienten und steht für die gebietsübergreifende Routine oder spezielle infektiologische stationäre Versorgung zur Verfügung. Mitarbeiterinnen und Mitarbeiter, die nicht in die internistische Versorgung integriert werden, bieten Einzelversorgung (Psychotherapie) per Videoschaltung oder Telefon an. Allerdings konnte bisher die Gruppenpsychotherapie videogestützt oder in Form einer Telefonkonferenz in der Kassenärztlichen Vereinigung Bayerns (KVB) und Bayerischen Krankenhausgesellschaft (BKG) politisch nicht durchgesetzt werden. Aus Sicht der BKG bestünde bezüglich der „Virtualisierung“ von Gruppenpsychotherapie explizit keine Einschränkung, da die Vergütung über den „Schutzschirm“, der mit der Bereitstellung von Betten für die COVID19-Therapie verbunden ist, pauschal erfolgt und inhaltlich nicht definiert ist. Versorgt werden in diesem Kontext nun die vor dem eigentlichen Abschluss der stationären Behandlung entlassenen Patientinnen und Patienten sowie diejenigen, die sich bereits für eine Aufnahme interessiert hatten („Warteliste“).
2. Psychosomatische Rehakliniken entlassen möglichst alle ihre Patienten, die entlassungsfähig sind und passen die Angebote für die noch verbleibenden Patienten hygienetechnisch an. Die Kliniken erhalten für die frei gehaltenen Betten $60 \%$ des Tagessatzes, können Kurzarbeit anmelden, Kredite der Kreditanstalt für Wiederaufbau (KfW) beantragen, aber auch im Rahmen der Allgemeinverfügung als Notkrankenhaus in die Versorgung einbezogen werden. Dann kann die Personalgestellung über die Zuarbeit des Pandemiearztes des Landkreises erfolgen (einschließlich Zwangsrekrutierung von ärztlichen oder pflegenden Kollegen, die bei Weigerung notfalls von der Polizei abgeholt würden, ähnlich wie bei einer Verhaftung).

3. Es gibt aber auch psychosomatische Kliniken, die sowohl im Rehabereich als auch in der Krankenhausversorgung unter Beachtung der Hygienemaßgaben und der daraus resultierenden veränderten therapeutischen Strategien mit reduzierter Patientenzahl weitermachen.

In den bayerischen Hochschulambulanzen dürfen Patientinnen und Patienten über alle Gebiete hinweg per Telefon und Videotherapie versorgt werden, um das unmittelbare Infektionsrisiko in den Kliniken zu reduzieren.

Es zeigt sich zunehmender Beratungsbedarf in Sachen Angst vor dem Coronavirus sowie zwischenmenschliche psychische und psychosomatische Probleme im Zuge der notwendigen sozialen Distanz (Pflegeheime). Das vorgehaltene Versorgungsangebot kann einiges an Problemen abfedern (bei bereits vorhandenen Ressourcen, die nicht anderweitig gebunden sind oder fehlen). Insbesonchosomatik in zumindest naher Zukunft 


\section{Psychotherapie aktuell}

\section{Tab. 1 COVID-19-Checkliste}

\begin{tabular}{|c|c|c|c|c|c|}
\hline \multirow[t]{2}{*}{ Nr. } & \multirow[t]{2}{*}{ Frage } & \multicolumn{2}{|c|}{14 Tage vor Aufnahme } & \multicolumn{2}{|c|}{ Zeitpunkt der Aufnahme } \\
\hline & & Ja & Nein & Ja & Nein \\
\hline 1 & Hatten Sie oder haben Sie heute Halsschmerzen? & $\square$ & $\square$ & $\square$ & $\square$ \\
\hline 2 & Haben/hatten Sie heute Husten? & $\square$ & $\square$ & $\square$ & $\square$ \\
\hline 3 & Haben/hatten Sie heute Fieber? & $\square$ & $\square$ & $\square$ & $\square$ \\
\hline 4 & Haben/hatten Sie heute Fieber gemessen? & $\square$ & $\square$ & $\square$ & $\square$ \\
\hline 5 & Haben/hatten Sie heute ein schweres Krankheitsgefühl? & $\square$ & $\square$ & $\square$ & $\square$ \\
\hline 6 & Haben Sie Angst, selbst einkaufen zu gehen? & $\square$ & $\square$ & $\square$ & $\square$ \\
\hline 7 & $\begin{array}{l}\text { Bemerken Sie neuerdings oder hatten Sie Schwindel oder andere } \\
\text { Symptome des Nervensystems? }\end{array}$ & $\square$ & $\square$ & $\square$ & $\square$ \\
\hline 8 & Bemerken Sie neuerdings oder hatten Sie Geruchsverlust? & $\square$ & $\square$ & $\square$ & $\square$ \\
\hline 9 & Bemerken Sie neuerdings oder hatten Sie Geschmacksverlust? & $\square$ & $\square$ & $\square$ & $\square$ \\
\hline 10 & Haben Sie eine Bindehautentzündung (Augen)? & $\square$ & $\square$ & $\square$ & $\square$ \\
\hline 11 & Lassen Sie sich Lebensmittel von anderen einkaufen? & $\square$ & $\square$ & $\square$ & $\square$ \\
\hline 12 & Gibt es in Ihrem Haushalt Personen, die jünger als 16 Jahre sind? & $\square$ & $\square$ & $\square$ & $\square$ \\
\hline 13 & Gibt es in Ihrem Haushalt Personen, die älter als 65 Jahre sind? & $\square$ & $\square$ & $\square$ & $\square$ \\
\hline 14 & Machen Sie sich Sorgen wegen Ihrer Gesundheit? & $\square$ & $\square$ & $\square$ & $\square$ \\
\hline 15 & Haben Sie sich aufgrund eines Virusverdachts testen lassen? & $\square$ & $\square$ & $\square$ & $\square$ \\
\hline 16 & $\begin{array}{l}\text { Haben Sie körperliche Krankheiten, z. B. Bluthochdruck, Zuckerkrank- } \\
\text { heit, Lungenerkrankungen? }\end{array}$ & $\square$ & $\square$ & $\square$ & $\square$ \\
\hline \multicolumn{6}{|c|}{ COVID-19 "coronavirus disease 2019" } \\
\hline
\end{tabular}

dere im Fall prekärer Wohnsituationen von Patienten ist aufgrund der oft mangelnden Privatsphäre und mangelnden technischen Ausstattung die Behandlung per Telefon leichter möglich als per Video. Auch die Nachfrage nach flankierender Betreuung der mit dem Thema „coronavirus disease 2019“ (COVID-19) Beschäftigten nimmt zu. Hier wurden bereits einige Projekte auf den Weg gebracht: zum einen konzertiert (in den meisten Universitätsklinika), wie z. B. als Mitarbeiterbefragung (Projektbeschreibung unter https://www.psychosomatik. uk-erlangen.de/forschung/) aber auch als Einzelinitiativen etwa im Rahmen der Nationalen Kohortenstudie (from COVID-19 to COVAD-20 ["CoronaAnxiety Disorder"] oder "COVID19 Patient(inn)en zu VERSORGEN birgt besondere psychische Belastungen: VORBEUGEN ist möglich!“" (Braun und Loew, Dt. Ärzteblatt, im Druck).

\section{Welche Szenarien kommen auf die Patienten zu?}

Grundsätzlich wird versucht, die Kliniken "Coronavirus-frei“ zu halten, d.h., dass den Patientinnen und Patienten
14 Tage vor Aufnahme in die Klinik die in - Tab. 1 aufgeführten Fragen gestellt werden. Sind die Patientinnen und Patienten zu diesem Zeitpunkt infektionsfrei, kommen sie in die nähere Auswahl. In der Mehrzahl der Fälle erfolgt die Aufnahme nach weiteren 14 Tagen. Trifft die Patientin bzw. der Patient in der Klinik ein, stellt eine Kollegin, die die persönliche Schutzausrüstung (PSA) trägt, ihr oder ihm noch einmal dieselben Fragen. Seit Mitte März wurde zunehmend häufiger, in Bayern wird seit dem 8.5. auf Erlass des Gesundheitsministeriums hin nun obligat Material für einen Coronavirus-Nachweis entnommen (mithilfe eines Rachenabstrichs oder der tiefen Rachenspülung).

Die Patientinnen und Patienten begeben sich bis der aktuelle Befund vorliegt in eine „Kurzquarantäne“, in der sie behandelt werden, als ob sie infektiös wären (d.h. wenige Stunden, in der Regel 2). Danach werden sie ...

1. bei negativem Befund zu "normalen“ Patienten oder

2. bei positivem Befund, wenn keine unmittelbare medizinischen Behandlungsnotwendigkeit besteht und die Entlassung möglich ist, in die häusliche Quarantäne entlassen oder,

3. wenn es die Ausprägung und Art der medizinischen Probleme erfordert, auf eine Infektionsstation verlegt.

4. Ist der Befund positiv, aber aus psychosomatischer Sicht eine medizinisch notwendige Behandlung erforderlich, erfolgt eine angepasste Behandlung in Klinikquarantäne (wenn dies mehrere Patientinnen und Patienten betrifft, auf einer speziellen Coronavirus-Station). Hierbei muss die/der Betroffene ähnlich wie in der häuslichen Quarantäne verbleiben.

Letzteres stellt für alle Beteiligten die größte Herausforderung dar. Von großem Vorteil ist es, wenn das Patientenzimmer einen Balkon hat. Dieser kann etwa für „Draußen“-Sport und -Bewegung genützt werden, auch mit Anleitung durch den/die Physiotherapeuten/ Physiotherapeutin, der/die z.B. unten vor dem Haus steht. Materialien zum kreativen Arbeiten können aufs Zimmer gebracht werden; die Anleitung kann per Videoverbindung oder Telefon erfolgen, wenn es der oder dem Patienten möglich ist, über diesen Weg eine Bindung 
aufzubauen. Ausgewählte Filme oder Bücher/Hörbücher können den psychotherapeutischen Prozess unterstützen. Auch die Angehörigen können über diesen Weg einbezogen werden. Muss der Therapeut im Zimmer arbeiten, ist dies nur beim Tragen der entsprechenden PSA vertretbar.

\section{Welche allgemeinen Hygieneregeln gelten aus psychosomatischem Blickwinkel?}

Die Vorgehensweisen wurden von professionellen Teams entwickelt und entsprechend überprüft. Diese setzten sich zusammen aus den jeweiligen Hygienefachkräften (besonders qualifizierte Krankenpfleger oder Krankenpflegerinnen), die vollschichtig in den Einrichtungen beschäftigt sind, besonders weitergebildeten Ärzten, die zumindest stundenweise in der jeweiligen Klinik beschäftigt sein müssen, und den jeweiligen Chefärzten.

Grundsätzlich können und müssen wenigstens an einigen Standorten auch "Coronavirus-positiv getestete“ Patientinnen und Patienten behandelt werden. In vielen psychosomatischen Kliniken in Bayern besteht der Vorteil, dass die Patientinnen und Patienten von vornherein Einzelzimmer belegen. In der Isolation ist es aber auch, wie in den internistischen „Coronavirus-Einheiten“ möglich, mit Mehrfachbelegung zu arbeiten, da die Patientinnen und Patienten bereits nachweislich infiziert sind. In den meisten Fällen gesunden diese Patientinnen und Patienten auch innerhalb von 14 Tagen und entwickeln wahrscheinlich eine spezifische Immunität, so lautet zumindest die Meinung der meisten Virologen und Immunologen weltweit.

Es empfiehlt sich die räumliche Konzentration, wenigstens auf einem Stockwerk. Die Pflegekräfte erledigen einmal täglich alle bei diesen Patienten notwendigen Arbeiten „in einem Rutsch“. Dabei tragen sie Einmalkittel, Handschuhe und Mund-Nasen-Schutz, der während der Tätigkeiten nicht abgesetzt wird. Der Mund-Nasen-Schutz wird danach verworfen. Die Patienten werden mit einem Mund-Nasen-Schutz versorgt und ange-

Psychotherapeut 2020 -65:285-290 https://doi.org/10.1007/s00278-020-00426-w

(c) Springer Medizin Verlag GmbH, ein Teil von Springer Nature 2020

\section{T. H. Loew $\cdot$ M. Krinninger $\cdot$ C. Kettler}

\section{Stationäre Psychosomatik in Zeiten des Coronavirus}

\section{Zusammenfassung}

Die aktuelle Coronavirus-Pandemie stellt psychosomatische Kliniken vor neue Herausforderungen. Damit auch niedergelassene Psychotherapeuten sich für ihre Patientinnen und Patienten ein Bild machen können, wurden im Folgenden zentrale hygienische Aspekte aufbereitet. Drei grundsätzliche Versorgungsmuster und Angebote der Einrichtungen können unterschieden werden: Umwidmung, schrittweise Räumung und Stand-by für die Umwidmung sowie Fortsetzung des Betriebs unter seuchenmedizinischen Rahmenbedingungen. Inhaltlich werden folgende Themen relevant: Beratungsbedarf in Sachen Angst vor dem Coronavirus sowie zwischenmenschliche psychische und psychosomatische Probleme im Zuge der notwendigen sozialen Distanz, Versorgung insbesondere bei prekären Wohnsituationen, veränderte Kommunikationsstrukturen (Telefon, Videosprechstunde) und Betreuung der mit dem Thema "coronavirus disease 2019“ (COVID-19) Beschäftigten. Alle Patientinnen und Patienten durchlaufen vor der
Aufnahme einen klinischen und virologischen Diagnoseprozess und werden, evtl. unter Quarantänebedingungen beginnend, psychosomatisch-psychotherapeutisch stationär versorgt. Abschließend wird dargestellt, was den Patientinnen und Patienten mithilfe eines einfachen Merkschemas mitgeteilt wird: Abstand (mindestens $2 \mathrm{~m}$ ), Abreibung (oft und wenigstens $20 \mathrm{~s}$ lang Händewaschen mit Seife), Ausweichen (freundliches Aneinandervorbei-Gehen), alternative Kommunikation, Aussetzen (es geht auch ohne Shopping), Abwischen (regelmäßig alle Flächen, durch das Reinigungspersonal, aber auch Tablet und Handy durch die Patientin oder den Patienten) und möglichst außen bewegen, allein oder zusammensitzen (mit Sicherheitsabstand). Das psychosomatische Angebot wurden entsprechend umgewandelt.

\section{Schlüsselwörter}

"Coronavirus disease 2019" . Patientenversorgung · Persönliche Schutzausrichtung . Hygiene - Check-Liste

\section{Inpatient psychosomatics in times of the coronavirus}

\section{Abstract}

The current coronavirus 2019 (COVID19) pandemic presents psychosomatic clinics with new challenges. In order that psychotherapists in private practice can also obtain a picture for their patients, this article deals with the core aspects of hygiene. There are three fundamental patterns of care and provisions by the institutions that can be differentiated: rededication, stepwise evacuation and stand-by for rededication and continuation of the service under medical epidemic conditions. The following topics are the relevant contents: need for consultation in the matter of anxiety for coronavirus and interpersonal mental and psychosomatic problems due to the necessary social distancing, care especially in precarious living situations, altered communication structures (telephone, video consultation) and care of those occupied with the topic of "coronavirus disease 2019" (COVID-19). All patients undergo a clinical and virologic diagnostic process before admittance and receive psychosomatic psychotherapeutic inpatient care, possibly beginning under quarantine conditions. Furthermore, appropriate general hygiene regulations are explained. Finally, what the patients are told is illustrated using a simple schematic aid: distance (minimum $2 \mathrm{~m}$ ), rubbing (wash hands with soap often and for at least $20 \mathrm{~s}$ ), avoidance (pass by in a friendly manner), alternative communication, forsaking (shopping is not always necessary), wiping (regularly wipeing of all surfaces by the cleaning personnel as well as tablet and mobile telephone by the patients themselves) and outside activity as much as possible, alone or sitting together (with safety distance). The psychosomatic services were appropriately converted.

\section{Keywords}

Coronavirus disease 2019 . Patient care . Personal protective equipment . Hygiene . Checklist 
halten diesen aufzusetzen, solange die Pflegekraft im Raum ist. Auf die Handhygiene ist $\mathrm{zu}$ achten und generell stets so viel Abstand wie möglich zu halten. Gegessen wird auf den Zimmern. Das Essen wird vor dem Zimmer abgestellt die Patienten werden durch Anklopfen an der Tür hierüber informiert und angehalten, das Geschirr/Tablett vor der Tür abzustellen, wenn sie die Mahlzeit beendet haben. Von dort wird dieses abgeholt. Hierzu ist kein Mundschutz erforderlich. So sollte es möglich sein, dass pro Tag nur eine Pflegekraft im Kontakt mit den infizierten Patienten ist und nur ein Mund-Nasen-Schutz verbraucht wird, denn - wie der Presse zu entnehmen ist - der Nachschub von medizinischem Mundschutz stellt aktuell das Problem dar. Potenziell kontaminierter MundNasen-Schutz wird nicht mehr verwendet, was einer Verschleppung vorbeugen soll. Falls dringend ein weiterer Kontakt notwendig ist (z.B. bei einer Zustandsverschlechterung des Patienten), sollte ein weiterer Mund-Nasen-Schutz getragen werden, der ebenfalls im Anschluss weggeworfen wird.

Für die Behandlung nichtbeatmeter COVID-19-Patienten wird das Tragen von Mund-Nasen-Schutz zusätzlich zur allgemeinen PSA vorgeschlagen (Handschuhe, Kittel, Augenschutz z.B. durch Brille oder Visier; laut Leitlinien schwache Evidenz (https://www.rki. de/DE/Content/InfAZ/N/Neuartiges_ Coronavirus/Hygiene.html), entspricht "guter klinischer Praxis“). Die Desinfektionsregeln müssen beachtet werden. Die Patientin oder der Patient trägt mindestens einen chirurgischen MundNasen-Schutz und befolgt die Abstandsund Hygieneregeln.

Allerdings ist das Zusammenstellen von separaten Teams, die sich wie in der Infektiologie ausschließlich um die infizierten Patienten kümmern, nicht möglich, da das erforderliche Personal nicht verfügbar ist. Dies ist aber auch unter den gegebenen Umständen nicht zwingend erforderlich. Ziel ist es, dass die Coronavirus-positiven Patienten so schnell wie möglich (je nach klinischem Zustand) verlegt oder in Absprache mit dem Gesundheitsamt nach Hause entlassen werden. Hier wird sich an die
Vorgaben vom Gesundheitsamt zum Entlassungsmanagement (https://www.rki. de/DE/Content/InfAZ/N/Neuartiges_

Coronavirus/Hygiene.html) gehalten. Nach ersten Erfahrungen lehnt das Gesundheitsamt auch bei symptomarmen oder sogar symptomfreien Patienten eine Entlassung nach Hause über weitere Strecken $a b$, weil jederzeit eine rasche Verschlechterung der Symptomatik eintreten kann, was unterwegs (z.B. auf der Autobahn) fatal wäre. In solchen Fällen ist die Verlegung in ein Krankenhaus vorzuziehen. Alle Mitarbeiter, die mit Patienten arbeiten, sollten bis auf Weiteres ebenfalls durchgehend einen Mund-Nasen-Schutz tragen und diesen am Ende des jeweiligen Arbeitstages verwerfen - gleichgültig, ob sie näher an Patienten herantreten als 2 m oder nicht. Es sollten FFP2-Masken mit Filter zur Verfügung stehen, die besonders zum Eigenschutz dienen. Ihr Filter verhindert den Übertritt von Feinstoffen oder Viren und Bakterien zu 99\%. Diese sollten aber nur noch sehr sparsam benutzt werden, z. B. bei der Durchführung eines Abstrichs (und allen anderen aerosolbildenden Tätigkeiten). Entsprechend den Leitlinien werden die dicht sitzend angelegten FFP2-Masken (https://www.rki. de/DE/Content/InfAZ/N/Neuartiges_ Coronavirus/Hygiene.html) bei aerosolgenerierenden Maßnahmen zusätzlich zur allgemeinen PSA angewendet. Hierzu zählen Intubation, Bronchoskopie, offenes Absaugen, Vernebelung, manuelle oder nichtinvasive Beatmung, Diskonnexion des Beatmungssystems, Umlagerung von oder zur Bauchlagerung und Reanimation.

Im Zuge dessen sollte auch eine Schutzbrille oder ein Visier getragen werden. Die Erfahrung zeigt, dass das Visier, das selbst über der eigenen Brille getragen werden kann, von den Mitarbeiterinnen und Mitarbeitern bevorzugt wird. Es kann mehrfach verwendet werden und ist wesentlich leichter $\mathrm{zu}$ reinigen bzw. zu desinfizieren als die Brille. Mittlerweile drucken viele Kliniken die Halterungen für das Visier selbst aus (per 3D-Drucker; z. B. bei https:// www.prusaprinters.org/prints/25857protective-face-shield-rc1, Druckdauer ca. $3 \mathrm{~h}$ pro Haltevorrichtung, reine
Materialkosten ca. $2 €$, im Handel $24 €$ ). Als Schild wird z.B. Laminierfolie oder ähnliches Material verwendet. Die im Handel erhältlichen, zertifizierten Masken tragen entweder den Zusatz „NR“ (nichtrecyclebar) oder „R“ (recyclebar).

Benutzte FFP3-Masken und Visiere/ Schutzbrillen werden, mit dem Namen des Trägers versehen, einzeln in Plastiktüten gesammelt, da es notwendig werden könnte, diese personenbezogen aufzubereiten, falls das Material ausgeht. Hierbei ist es wichtig, dass die Tüte nicht verschlossen wird, damit sich auf den Masken kein Schimmel bildet.

Grundsätzlich sollten, wie bereits mehrfach betont, Tätigkeiten, die das Unterschreiten des Mindestabstands von $2 \mathrm{~m}$ erfordern, auf ein absolut notwendiges Mindestmaß beschränkt werden. Beispielsweise ist es aktuell vertretbar, die körperliche Untersuchung der Rehabilitanden weniger umfänglich durchzuführen und eher anlassbezogen $\mathrm{zu}$ untersuchen (z.B. bei Beschwerden oder wenn sozialmedizinische Fragen zu klären sind).

Eine Anmerkung zum Joggen oder Wandern im Freien: Hier zeichnet sich $a b$, dass der Mindestabstand größer als $2 \mathrm{~m}$ sein sollte. Insbesondere sollte niemand im Windschatten eines potenziell infektiösen Menschen laufen, da gerade bei forcierter Atmung während des Sports eine große Menge Aerosol die Lungen verlässt und eine Tröpfcheninfektion über eine größere Distanz als $2 \mathrm{~m}$ wahrscheinlicher wird.

\section{Was wird den Patientinnen und Patienten mitgeteilt?}

In einer psychosomatischen Klinik leben alle auf engem Raum zusammen, eigentlich einer Familie ähnlich oder vergleichbar. Wäre einer der anderen Patienten oder der Mitarbeiter oder deren Kontaktperson im persönlichen Umfeld nachweislich betroffen, würden die Menschen, die unmittelbaren Kontakt mit der Person hatten (den bekannten „Südtiroler $75 \mathrm{~cm}$ Abstand" für länger als $15 \mathrm{~min}$ unterschritten und dabei im Gespräch befindlich) in 14-tägige Quarantäne gehen müssen. Bei den Mitarbeitern, die unter Beachtung der Abstandsregel und 


\section{Infobox 1 Merkhilfe für Patientinnen und Patienten: 7 A(uf) einen Streich}

- Abstand $2 \mathrm{~m}$

- Abreibung (oft wenigstens 20 s Händewaschen, gern mit entschleunigtem Atmen und/oder Summen verbinden)

- Ausweichen (freundliches Aneinander-vorbei-Gehen, Lächeln ist nicht verboten)

- Alternative Kommunikation (soziale Medien, Telefon, Videokommunikation)

- Aussetzen (es geht auch ohne Shopping, Bars, Theater etc.)

- Abwischen (regelmäßig, d.h. nach jedem Gebrauch, bevor jemand Neues sie nutzt: alle Flächen, aber auch Tablet und Handy nicht vergessen) und möglichst

- Außen bewegen, allein oder zusammen sitzen (beachte: mit Sicherheitsabstand)

mit Mund-Nasen-Schutz arbeiten, würde gewartet, bis sie symptomatisch werden; erst dann würde die Quarantäne angeordnet, die wenigstens 7 Tage, viel eher 14 Tage oder bis zum Ende der Symptomatik aufrechterhalten würde.

Da das Virus sich über Tröpfcheninfektion verbreitet, gilt: Tröpfchen möglichst aus dem Weg gehen. „Feuchte Aussprache", Niesen und Husten sind das direkte Problem, das indirekte, wo die Tröpfchen überall landen können. Was dies für die Mitarbeitenden in der Klinik und außerhalb bedeutet, ist in $\bullet$ Infobox 1 zusammengefasst.

Tröpfchen beim Husten und beim Niesen sollten mithilfe eines Papiertaschentuchs aufgehalten werden, gefolgt von der Entsorgung des Papiertaschentuchs im geschlossenen Mülleimer. Häufig berührte Gegenstände und Oberflächen sollten regelmäßig mit einer Seifenlösung gereinigt und/oder desinfiziert werden. Nach dem Vorbild des Hongkonger Protokolls (https://www.chp.gov.hk/ files/pdf/nid_guideline_general_public_ en.pdf) wird den Bürgern empfohlen, jederzeit Gesichtsmasken zu tragen, wenn sie sich in Gegenwart anderer Personen befinden, um sich vor dem Virus zu schützen und es nicht zu verbreiten. Das COVID-19 auslösende Virus mit dem Namen SARS-CoV-2 kann $72 \mathrm{~h}$ (einige Virologen schätzen bis zu 7 Tage) auf Oberflächen außerhalb des Körpers überleben. Desinfektion ist daher entscheidend. Im Körper findet sich SARS-
CoV-2 hauptsächlich auf der Oberfläche von Geweben, die einen spezifischen Coronavirus-Rezeptor aufweisen (innerer Hals, Lungen).

Bis auf Weiteres sind in den Kliniken die sonst üblichen Belastungserprobungen im häuslichen Umfeld am Wochenende ausgesetzt, da das Infektionsrisiko verringert werden soll. Jeder Mensch, der einem anderen nahekommt, kann einen potenziellen Ansteckungsherd darstellen (auch wenn sie oder er selbst nicht krank erscheint). Bei Halsweh oder Husten und/oder Fieber ist doppelte Vorsicht geboten (als Betroffener und als Begegnender).

Gern dürfen die Patientinnen und Patienten außerhalb der Klinik spazieren gehen, das Aufsuchen von Orten, in denen sich andere Menschen aufhalten, sollte von den in - Infobox 1 zusammengefassten Verhaltensregeln bestimmt sein. Es scheint nicht so $\mathrm{zu}$ sein, dass Türkliniken oder metallische Flächen potenziell für die Übertragung des Virus infrage kommen können, wohl aber Plastikflächen oder Karton.

Die potenzielle Virusbelastung der Therapiegeräte und -gegenstände wird so gut wie möglich - durch regelmäßige Desinfektion reduziert. Auf Besuche von außen wird verzichtet.

In den verschiedenen Gruppen wird versucht, den gebotenen Mindestabstand von $2 \mathrm{~m}$ einzuhalten, d. h., dass Gruppengröße und -zeit halbiert werden. Angebote wie Tanztherapie oder Bewegungstherapien, in der/denen es manchmal „etwas enger“ werden kann, werden, wenn möglich, mit viel Zwischenraum gestaltet oder ausgesetzt. Stattdessen werden meditative Angebote, z. B. Gehmeditation, vorgehalten. Ergo- und Musiktherapie wird, wenn Materialien oder Gegenstände zum Einsatz kommen, auf Einzelanleitung oder -therapie umgestellt, um das Desinfizieren zu erleichtern. Sport-, Bewegungs- und Entspannungsgruppen werden ebenfalls, sobald es das Wetter erlaubt, ins Freie verlegt. Auch die Einzeltherapie kann durchaus in Form eines Spaziergangs oder in Form eines Telefonats erfolgen.

Frische Luft tut immer gut, auch wenn Ihnen „die Decke auf den Kopf“ zu fallen droht, und die umgebende Natur mit ihrem gesunden Klima, von dem im Klinikumfeld genug geboten werden kann, erledigt ihr Übriges. Sonnenlicht (UVStrahlung) hat mindestens eine gewisse (begrenzte) desinfizierende Wirkung, insbesondere auf das Coronavirus, v. a. bei trockenem Wetter. Für das Virus Covid P9, das SARS zugrunde liegt, liegen Daten vor, die belegen, dass dieses Virus nach 60 min UV Bestrahlung komplett zerstört ist (Duan et al. 2003).

Jede Krise beinhaltet auch eine Chance: Am besten haben Sie gelernt, sich $\mathrm{zu}$ regulieren, und dabei helfen Ihnen die Kinikmitarbeitenden.

\section{Abschließende Gedanken}

Besteht die Integration der psychosomatischen Abteilung in eine Klinik mit Intensivbetten, kann eine weitere Aufgabe der Psychosomatik auch die Mitarbeit in der klinischen Ethikkommission sein. Und ... was wäre ein Psychosomatiker, hätte er nicht auch in dieser Situation ein körpertherapeutische Angebot.

In den Nasengängen produziertes Stickstoffmonoxid ist Teil des Abwehrsystems gegen bakterielle und virale Infektionen. Es wurde z. B. festgestellt, dass Neugeborene einen extrem hohen Stickstoffmonoxidspiegel in ihren kaum entwickelten Nasennebenhöhlen haben. Einfaches Summen beim Ausatmen erhöht sofort die Stickstoffmonoxidkonzentration in den Nasengängen und Nebenhöhlen (Weitzberg und Lundberg 2002) - also genau in den Geweben, in denen SARS-CoV-2 viele Tage verweilt, bevor andere Gewebe infiziert werden. Zumindest bei den dem Menschen nahen Säugetierverwandten, den Schweinen, konnte ein direkter Zusammenhang zwischen hohen Stickstoffmonoxidspiegeln und einer spezifischen Reduktion von Coronaviren nachgewiesenen werden (Akerström et al. 2005). Den Mitarbeiterinnen und Mitarbeitern und auch den COVID-19-Patientinnen und -Patienten (zumindest denen, die nicht im künstlichen Koma sind) wird empfohlen zu summen. Nebenbei entschleunigt dies den Atemrhythmus und entstresst nachweislich (4 s Einatmen, 6 s Ausatmen; Loew 2019). Die Sauerstoffsättigung geht dabei nicht zurück, 
sondern steigt eher an (Sin et al. 2010), und viele Patientinnen und Patienten bestätigen aktuell, dass die subjektive Atemnot abnimmt. Was summen? Da gibt es keine Vorgaben: Youtube bietet viele Anregungen, ganz egal, ob „Geh aus, mein Herz, und suche Freud" vom Corona Virtual Choir, „Coronavirus Rhapsody“ oder "My Corona“. Also summen Sie, so oft Sie können, und Mund-Nasen-Schutz nicht vergessen.

\section{Korrespondenzadresse}

Dr. Maximilian Krinninger

Alpcura Fachklinik Allgäu Betriebsgesellschaft $\mathrm{mbH}$

Peter-Heel-Str. 29, 87459 Pfronten, Deutschland maximilian.krinninger@fachklinik-allgaeu.de

\section{Einhaltung ethischer Richtlinien}

Interessenkonflikt. T.H. Loew, M. Krinninger und C. Kettler geben an, dass kein Interessenkonflikt besteht.

Für diesen Beitrag wurden von den Autoren keine Studien an Menschen oder Tieren durchgeführt. Für die aufgeführten Studien gelten die jeweils dort angegebenen ethischen Richtlinien.

\section{Literatur}

Akerström S, Mousavi-Jazi M, Klingström J, Leijon M, Lundkvist A, Mirazimi A (2005) Nitric oxide inhibits the replication cycle of severe acute respiratory syndrome coronavirus. J Virol 79(3):1966-1969

Duan SM, Zhao XS, Wen RF, Huang JJ, Pi GH, Zhang SX, Han J, Bi SL, Ruan L, Dong XP (2003) SARS Research Team. Stability of SARS coronavirus in human specimens and environment and its sensitivity to heating and UV irradiation. Biomed Environ Sci 16(3):246-255

Loew TH (2019) Entschleunigtes Atmen. Psychother Dialog 18(04):63-67

Sin PY, Webber MR, Galletly DC, Ainslie PN, Brown SJ, Willie CK, Sasse A, Larsen PD, Tzeng YC (2010) Interactions between heart rate variability and pulmonary gas exchange efficiency in humans. ExpPhysiol 95(7):788-797

Weitzberg E, Lundberg J (2002) Humming greatly increases nasal nitric oxide. Am J Respir Crit Care Med 166:144-145

Alkoholbedingte Immunreaktion im Gehirn erhöht Suchtrisiko

Ein internationales Forscherteam unter Beteiligung des Zentralinstitut für Seelische Gesundheit (ZI) hat einen neuen Mechanismus entdeckt, wie Alkohol das Gehirn verändert. Andauernder Alkoholkonsum aktiviert hirneigene Abwehrzellen, sogenannte Mikroglia, was zu einer erhöhten Diffusion von Botenstoffen zwischen den Nervenzellen führt.

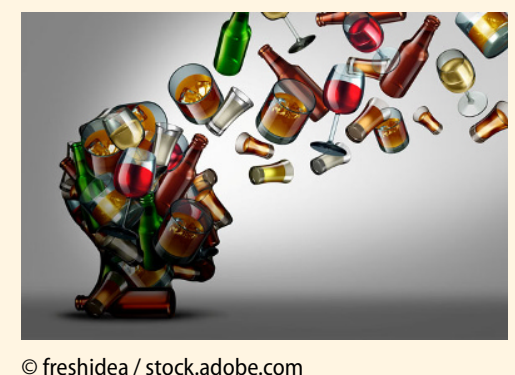

Wer Alkohol konsumiert, weiß in der Regel, dass zu viel des Guten süchtig machen kann. Was dabei im Gehirn passiert, ist aber weiterhin nicht klar. Ein Forscherteam aus vier europäischen Ländern unter maßgeblicher Beteiligung des Zentralinstituts für Seelische Gesundheit (ZI) in Mannheim hat nun in der Fachzeitschrift Science Advances über einen bisher unbekannten Mechanismus der Alkoholwirkung berichtet. Die Forscher beobachteten, dass Alkohol beim Menschen als auch bei Versuchstieren in der grauen Hirnsubstanz die Form und Struktur des Extrazellularraumes (EZR) verändert. Sie führen dies auf die Aktivierung von spezifischen Immunzellen des Gehirns, der Mikroglia, zurück.

\section{Immunzellen ziehen sich zurück}

Der EZR besteht aus Hohlräumen und Kanälen, die sich zwischen Nerven- und Gliazellen sowie deren vielen Fortsätzen bilden. Der EZR ist mit Flüssigkeit gefüllt. Dort zirkulieren viele Substanzen, die für verschiedene physiologische Prozesse notwendig sind. „Nach chronischer Alkoholexposition reagieren die Immunzellen des Gehirns, sie schrumpfen und ziehen ihr dichtes Geflecht aus Fortsätzen zurück. Durch den Wegfall von Barrieren ändert sich die Geometrie des EZR und es ergeben sich neue Diffusionswege. Viele Botenstoffe, wie zum Beispiel das für das Belohnungslernen wichtige Dopamin, verteilen sich über das Volumen des EZR. Die erhöhte Diffusion kann ihre Aktivität deutlich beeinflussen", erklärt Dr. Santiago Canals vom Instituto de Neurosciencias in Alicante/Spanien, der gemeinsam mit Prof. Dr. Wolfgang Sommer, stellvertretender wissenschaftlicher Direktor des Instituts für Psychopharmakologie und Oberarzt an der Klinik für Abhängiges Verhalten und Suchtmedizin am Zl, für die Studie verantwortlich ist.

\section{Volumentransmission steigt an}

Wenn die Diffusion im EZR erhöht ist, dann steigt auch die sogenannte Volumentransmission. Diese ist eine besondere Art der Signalübertragung im Gehirn. Sie unterscheidet sich von der üblichen Punkt-zu-PunktKommunikation über Synapsen durch das gleichzeitige Erreichen vieler Kommunikationselemente über die in den EZR freigesetzten Neurotransmitter. „Erhöhte Diffusion im EZR und Volumentransmission mögen als sehr unspezifische Wirkmechanismen für eine Droge erscheinen. Dadurch werden aber eine Vielzahl von Kommunikationsprozessen im Gehirn beeinflusst", sagt ZIForscher Prof. Dr. Wolfgang Sommer. „Die in unserer Studie beschriebenen Interaktionen zwischen Nerven- und Immunsystem bieten einen Erklärungsansatz, wie Alkohol, trotz anfänglich recht schwacher akuter Effekte auf das Belohnungssystem, über die Zeit Anpassungsreaktionen auslöst, welche seine Wahrnehmung und seinen Konsum begünstigen sowie das Verlangen nach der Droge steigern, mit anderen Worten eine Suchtentwicklung befördern," ergänzt Prof. Sommer.

Originalpublikation: "Chronic alcohol consumption alters extracellular space geometry and transmitter diffusion in the brain", Science Advances. 2020. DOI: 10.1126/sciadv.aba0154

Quelle: Zentralinstitut für Seelische Gesundheit (ZI): www.zi-mannheim.de/forschung.html [24.06.2020] 